\title{
Design Methodology for Wireless Nodes with Printed Antennas
}

\author{
Jean-Samuel Chenard \\ jsamch@macs.ece.mcgill.ca \\ Željko Žilić \\ zeljko@macs.ece.mcgill.ca \\ Chun Yiu Chu ${ }^{\dagger}$ \\ cchu@cadlab.ece.mcgill.ca \\ Milica Popović \\ poppy@ece.mcgill.ca \\ Department of Electrical and Computer Engineering
McGill University, Montréal, Québec, Canada
}

\begin{abstract}
The need for mass-produced inexpensive wireless devices operating under strict energy constraints poses new challenges in the system design methodology. This paper presents a methodology for designing wireless nodes in which a low cost, reliable antenna is realized by printed circuit traces. We show how to combine the analysis from 2.5D and 3D EM simulators with the PCB design tools to create predictable nodes with printed antennas that meet stringent power and data transmission range goals. The presented approach is applied to the design of a IEEE802.15.4 wireless node deployed in several indoor environments.
\end{abstract}

\section{Categories and Subject Descriptors}

I.6.5 [Simulation and Modeling]: Model Development - Antennas; J.2 [Physical Sciences and Engineering]: Electronics - Wireless Communications; J.6 [Computer Applications]: Computed-Aided Engineering — Electromagnetic simulation

\section{General Terms}

Theory, Design, Experimentation, Measurement

\section{Keywords}

RF CAD, Printed Antenna, Antenna Design Methodology, Printed Circuit Board

\footnotetext{
*Microelectronics and Computer Systems Laboratory

${ }^{\dagger}$ Computational Electromagnetics Software Laboratory
}

Permission to make digital or hard copies of all or part of this work for personal or classroom use is granted without fee provided that copies are not made or distributed for profit or commercial advantage and that copies bear this notice and the full citation on the first page. To copy otherwise, to republish, to post on servers or to redistribute to lists, requires prior specific permission and/or a fee.

DAC 2005, June 13-17, 2005, Anaheim, California, USA

Copyright 2005 ACM 1-59593-058-2/05/0006 ...\$5.00.

\section{INTRODUCTION}

Recent advances in CMOS integrated circuits have allowed the integration of an RF front end onto the same die as the digital demodulation and media access control components. The high level of integration allows designers to add wireless networking capabilities to many embedded systems for a few dollars in additional costs. One of the major cost factor in adding RF capability to a product are the antenna, $\mathrm{RF}$ connectors and associated interface circuits. Since their combined price can often exceed that of the transceiver IC, the prospect of their elimination is appealing.

This paper develops a methodology for physical design of nodes for Wireless LANs, Wireless Sensor Networks (WSNs), or Personal Area Networks (PANs) where an efficient antenna is an integral part of the printed circuit board (PCB), thereby removing the cost of this component at the expense of some PCB area, and the careful design methodology that avoids interference with the rest of the system.

Further benefits of printed antennas include inherent ease in manufacturing and reliable wireless nodes. Antenna connectors and external antennas are notoriously fragile mechanical parts that often require protection against electrostatic discharge (ESD) as well as the additional steps in the system manufacturing. Throughout the node lifetime, thermal cycling will not affect the printed antenna, unlike, e.g., ceramic-based chip antenna whose thermal expansion coefficients is hard to match with the PCB. The integration of the antenna also results in a thin form factor, which is becoming important in an increasing number of applications.

As wireless networking nodes are often powered by small batteries, the efficiency of the antenna is of tantamount concern. A good antenna will save energy, by allowing reduced energy emission for the same distance. Conversely, a $3 \mathrm{~dB}$ loss in the antenna structure will require doubling the transmit power to achieve the same range while preserving the same Bit Error Rate (BER). Further, for a given transceiver energy emission, the improved signal level due to a good antenna will cause the decrease in BER and, consequently, less retransmissions, which further reduces energy consumption. Increased range will also provide system-level cost savings by reducing the required node density. 
The cost of the printed antenna is primarily determined by the board area that it occupies. The antenna should thus be made as small as possible. This constrains the antenna topology to monopoles or dipole strip antennas. Since the manufacturing of PCBs is optimized for mass production, it is required that the fabrication of the printed antennas does not modify the regular manufacturing process and be robust to etching inaccuracies.

This paper reviews the basic concepts required to design the printed antenna nodes in Section 2. Section 3 details the design methodology and its integration in the PCB design flow, along with the issues encountered while integrating the antenna with the remaining circuitry. The methodology is applied to the design of a IEEE 802.15.4 sensor node using a single chip CMOS transceiver and a printed dipole antenna in Section 4. The results are presented in Section 5.

\section{BACKGROUND}

In wireless devices, antenna is a structure responsible for the transfer of energy from PCB wires (a guided electromagnetic wave) to the free-space electromagnetic wave and vice versa. While any $\mathrm{PCB}$ trace will emit and receive some energy from free space, and cause a variety of Electromagnetic Interference (EMI) problems, good antennas will facilitate efficient communication at a distance, while not affecting the rest of the system to which it is attached.

In the design of wireless systems, the physical range forces major decisions in early design stages. A common scenario assumes starting with the known transmitter power $P_{t}$ and the receiver sensitivity $P_{r}$ and reaching the antenna gain values that guarantee a given physical range. The "best case" estimate for characterizing the range is based on the Friis Transmission formula for free space [11]:

$$
\begin{aligned}
P_{r}(d B m)= & P_{t}(d B m)+G_{t}(d B)+G_{r}(d B) \\
& -20 \log R(k m)-20 \log f(M H z)-32.44
\end{aligned}
$$

Transmitter gain $G_{t}$ and receiver gain $G_{r}$ can be obtained from antenna datasheets, measurements or, in the case of printed antennas, directly from the electromagnetic (EM) radiation analysis. The received power must be discounted by the estimated losses in the antenna interface circuits, such as balanced-to-unbalanced (balun). The distances are reduced in practice by unpredictable factors such as multipath dispersion, scattering, polarization mismatches and noise that will be inevitably present in a real-world environment. For example, a concrete wall can reduce power by 8 to $15 \mathrm{~dB}[8$, p.159]. Therefore, at the system-level, in the absence of an accurate analysis of the environment, a good antenna will provide much needed headroom, especially if the communication is power-limited.

\subsection{Operating Frequency and Bandwidth}

Most wireless applications use the instrumentation, scientific and medical (ISM) frequency bands that do not require the band-usage license. Specifically, the $2.4 \mathrm{GHz}$ ISM band offers good tradeoffs for range and overall antenna size. This band has a center frequency $f_{C}$ at $2.45 \mathrm{GHz}$ and spans frequencies from $f_{L}=2.4 \mathrm{GHz}$ to $f_{U}=2.5 \mathrm{GHz}$. The relative bandwidth $B_{p}$ required by the antenna is bounded by:

$$
B_{p}=\frac{f_{U}-f_{L}}{f_{C}} \times 100 \%
$$

In this case, $B_{p}$ is at most $4.1 \%$, which is best served by the class of narrow-band antennas [11].

Resonant antennas act by effectively lowering their impedance over a relatively narrow frequency band, and are our primary narrow-band antenna choice. This class of antennas also has an advantage from the standpoint of Electromagnetic Compatibility (EMC). The resonant antenna will reject the interference coming from the rest of the system and will not exacerbate the EMI emissions present in other parts as long as the system clocks on a board are relatively apart from the center frequency $f_{C}$. Indeed, this is the case in practice, where common microcontrollers run at clock rates that are orders of magnitude lower.

\subsection{Directivity, Gain and Efficiency}

The directivity of the antenna is defined as the ratio of power density in the direction of the pattern maximum to the average power density at the same distance from the antenna. The gain is defined as the directivity reduced by the losses in the antenna structure. Efficiency is defined as the ratio of total radiated power to the net power accepted by the antenna.

Printed resonant antennas are inherently efficient in the $\mathrm{GHz}$ range since they are "electrically large"(their physical size is large when compared to the wavelength) [11] and the losses in the dielectric and antenna element are low. The key measure for the efficiency is the antenna gain, which quantifies the peak transmitted power density and thus addresses the issue of power limits imposed by standard regulations. The peak gain of the antenna is critical for the node placement in a given system. Therefore, the gain should be considered early on in the design process as it will strongly impact the system-level efficiency for a given topology. High antenna gain implies long reach for a minimum of invested energy, but also limits the angular coverage. Conversely, an omnidirectional radiation pattern imply a low gain. This motivates us to accurately predict antenna gain considering losses in its structure, as well as in the casing of the wireless node.

We are mostly interested in the far-field (sometimes called Rayleigh distance) values of the gain and other antenna parameters. The far field conditions are given by the following conditions:

$$
r>\frac{2 D^{2}}{\lambda} ; r \gg D ; r \gg \lambda
$$

where $r$ is the distance to the antenna, $D$ is the maximal antenna dimensions and $\lambda$ is the wavelength.

\subsection{Polarization}

As a link between the free-space and guided waves, antenna is characterized by the way in which it radiates the electric field (E-field) and the magnetic field (H-field) [11]. These two components are inseparable and oscillate in a plane that is perpendicular to the direction (axis) of their propagation. Polarization of the antenna is defined by the geometric pattern that the emitted oscillating E-field vector tip traces in time. Polarization can be linear, circular or 
elliptic. The efficient receiving antenna should maximally match the polarization of the incoming waves (and, consequently, of the transmitting antennas) for best efficiency.

For printed antennas, we primarily consider linearly polarized antennas aligned with the PCB plane as, in practice, they allow for physically smaller structures than those possible for the case of circular or elliptical polarization. In some wireless networks, the orientation of the node cannot be controlled, hence the matching between the incoming waves and the reception antennas cannot be guaranteed. Linearly polarized printed antennas can still be efficient for the cost-sensitive nodes, if the hub nodes use circular polarization or electronically controllable polarization [3], often called "smart antennas". The main tradeoff for the use of circular polarization is a loss of at least $3 \mathrm{~dB}$ as a result of the mismatch between the circular and the linearly polarized antennas.

\subsection{Impedance and Matching}

For optimal power transfer between the transceiver and the antenna, their impedances must be matched. Computing the impedance and matching of the antenna port can be done analytically and then validated using the simulator. However, accurate extraction and simulation procedures are needed to estimate the real PCB trace impedances once the antenna is laid on the board. To simplify testing of antenna prototypes and allow in-system probing of the RF signal output, the RF path should preferably include the footprint for a $50 \Omega$ test connector in the microstrip line leading to the antenna.

For example, the IEEE 802.15.4 WLAN transceiver [2] used has a differential output of $115+\jmath 180 \Omega$. A balun network [2, p.19] has to be used to convert the impedance to a $50 \Omega$ single ended connection so that a standard SMA connector can be used to measure the transceiver output characteristics, balun losses and antenna admittance $\left(S_{11}\right)$ properties.

\subsection{PCB Material Limitations}

Many different materials are used for PCBs, including the compressed cardboard impregnated with phenolic resin and the high-end ceramic or Teflon based materials. The most common material is the inexpensive fiberglass-based FR4, with good mechanical and dielectric properties. FR4 can be used for antennas operating in the range of $2 \mathrm{GHz}$ - better materials are needed for more predictable high-frequency antennas.

The dielectric constant $\left(\epsilon_{r}\right)$ and loss tangent $(\tan \delta)$ of the PCB material have a notable impact on the printed antenna performance and the integration in the product. The antenna dimensions depend heavily on $\epsilon_{r}$ and the trace length needed for a resonant antenna is proportional to $1 / f \sqrt{\epsilon_{r}}$. For small nodes, some dielectric materials have $\epsilon_{r}$ values as large as $\epsilon_{r}=10$, which significantly reduces the printed antenna area. As it is of fundamental importance to narrowband resonant antennas, it is critical that $\epsilon_{r}$ remain controlled in the course of antenna fabrication.

High-frequency dielectrics posses higher uniformity of $\epsilon_{r}$ than FR4, especially for large temperature variations. The characteristics of common materials at room temperature and their tolerance are highlighted in Table 1. Using FR4 as the antenna material is thus possible, provided that some control on $\epsilon_{r}$ guarantees consistency between lots and that temperature extremes are considered in the simulation.

The dielectric loss tangent $\tan \delta$ primarily affects the antenna efficiency. High values of $\tan \delta$ imply that some power will be lost in the dielectric, reducing the gain of the antenna. In Table 1 one can note the 10-fold increase in loss tangent of FR4, compared to a high-frequency PCB material. In simulation models, this increase in the loss tangent translates to a loss of antenna efficiency of $\sim 0.4 d B$. Since the power emitted by nodes operating in the ISM bands is low, the loss of $0.4 \mathrm{~dB}$ is acceptable, as FR4 provides cost savings.

Table 1: PCB Materials Electrical Properties

\begin{tabular}{|c|c|c|c|}
\hline Material & Tan $\delta$ & $\epsilon_{r}$ & $\epsilon_{r}$ tol \\
\hline FR4 & 0.020 & $4.2-4.8$ & $13.6 \%$ \\
\hline RO4350B & 0.0031 & 3.48 & $2.8 \%$ \\
\hline RO3006 & 0.0020 & 6.15 & $4.8 \%$ \\
\hline RO3010 & 0.0023 & 10.2 & $5.8 \%$ \\
\hline
\end{tabular}

Printed microstrip trace impedance is given by the following expression:

$$
Z_{0}=\frac{87}{\sqrt{\epsilon_{r}+1.41}} \ln \left(\frac{5.98 H}{0.8 W+T}\right)[\Omega]
$$

Where the notation for the geometric parameters of the trace (given in mils) above the ground plane are as follows: W - width, $\mathrm{H}$ - height (thickness of the dielectric), and $\mathrm{T}-$ thickness the copper trace.

Often, PCBs are 0.062" (1.57mm) thick. Through experimentation, it was found that a dielectric thickness of 0.030 " $(0.762 \mathrm{~mm})$ was the best compromise when antennas were fabricated for a frequency of operation of $2.4 \mathrm{GHz}$. Thicker PCB material increased the size of $50 \Omega$ traces, the polarization mismatch and the consequent power waste. Thinner PCB material made the antennas fragile or not sufficiently rigid.

\section{DESIGN METHODOLOGY OVERVIEW}

The design flow used for our wireless node is shown in Figure 1. The initial step was to limit our search to relatively omnidirectional antennas with linear polarization. Throughout the process, it is unfeasible to apply the full three-dimensional (3D) modeling of the E- and H-fields, together with their abrupt changes and coupling to the rest of PCB. The first design iterations are performed on the $2.5 \mathrm{D}$ electromagnetic (EM) simulator to quickly characterize the antenna. Accurate modeling of the rest of the board is not yet required. Hence, all the details of the electronics are removed, except for the bottom ground plane required for modeling its effect on the antenna. The microstrip feed line is extended to reach the edges of the board such that an endlaunch SMA connector can be mounted as a test port. When simulations confirm the validity of the antenna, a PCB is etched using rapid prototyping methods. Etching accuracy is not too important as an inaccuracy of $0.5 \mathrm{~mm}$ in the length of the half wave dipole will translate in a frequency shift of $\sim 17 \mathrm{MHz}$. PCB processes achieve much higher accuracy.

Lab measurements are next performed on the test antenna to ensure that all the modeling is correct and that no unexplained mismatch exist between the lab results and the 


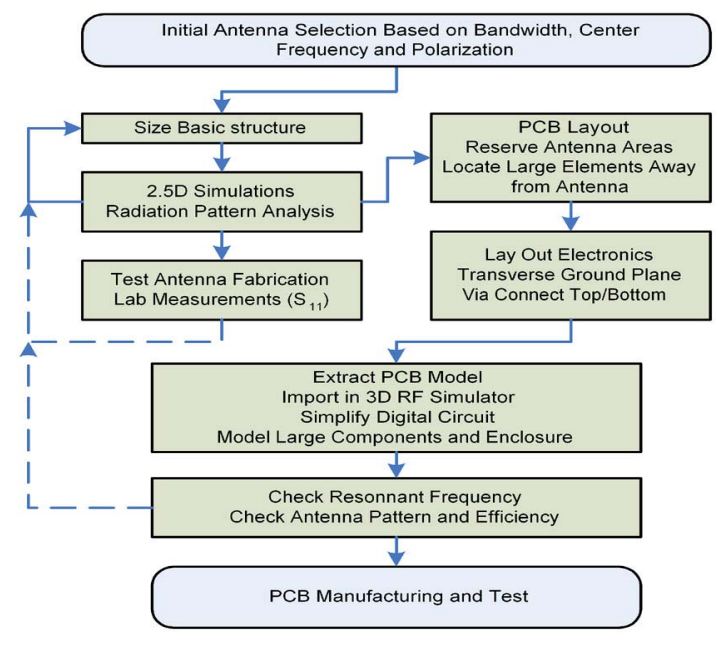

Figure 1: Printed antenna design flow

simulation model. The use of specialized RF laminates in general shows excellent correlation between lab results and simulation models, but this step is critical if the material like FR4 is used to ensure a good fit with the simulation. At that point, the PCB layout for the rest of the board can include the simplified antenna model in the PCB database. The antenna is at this stage given as a PCB route and plane obstruct zone constraint, to be replaced later with the final model.

As the PCB layout is nearing completion, it is extracted and prepared for a full 3D EM simulation, yielding in a more precise analysis. At this point, battery and enclosure models may be inserted into the $3 \mathrm{D}$ model to ensure that their presence in the design does not "de-tune" the antenna and to characterize their effect on the radiation pattern. When the antenna model is imported in the 3D EM simulator, digital lines and other non-RF signals can be removed since they do not affect the antenna and doing so will significantly speed up simulations. This extraction must preserve the via connections that link the top and bottom planes near the antenna and feed line.

\subsection{The 2.5D EM Simulator and its Limita- tions}

A 2.5D simulator is useful for planar and printed antenna designs. By using perfectly conducting strip layers (that have zero thickness and infinite conductivity) and infinitesized (but finite thickness) substrate layers, it provides fast and moderately accurate results. Agilent's Momentum ADS (v.2003A) is an example of a 2.5D simulator based on the Method of Moments (MOM) [1]. The antenna layout is best tested in range 1-3 GHz, resolving the shortest effective wavelength of the model with at least 30 cells of the numerical model mesh. To examine the effects of the metal thickness, the same antenna is re-simulated with copper of high (but finite) conductivity and the real thickness. The only observable difference should be a minor decrease of the gain. The decreased efficiency is due to the finite conductance of the copper leading to resistive losses.

\subsection{Simulation Using the 3D EM Simulator}

A 3D EM simulator can solve electromagnetic problems of arbitrary geometry, however, it demands larger computational resources. HFSS (v.9.2.1, Ansoft Corporation) simulator is based on the Finite-Element Method (FEM). As previously mentioned, a $3 \mathrm{D}$ version of the design is validated later in the design flow due to the longer run times. When the 3D model is re-simulated using HFSS, the space discretization of the dielectric substrate is based on subdividing a finite box instead of a $2 \mathrm{D}$ infinite plane. In this way, the transition from the substrate to the air will be considered more accurately. Generally, 3D full-wave simulations are prohibitively time-consuming. To reduce the simulation time, one can use the perfect conductor (infinite conductivity, zero thickness) instead of the real copper to outline the antenna structure.

\subsection{Modeling Comparison}

For the perfectly conducting antenna, results for the return loss obtained by Momentum and HFSS demonstrate a good match. The approximation of the metal with the perfect conductor becomes inaccurate for high frequencies $(>1 \mathrm{GHz})$ due to the presence of the skin effect.

The main difference between the two simulators is how they calculate the effects of the substrate layer. In $2.5 \mathrm{D}$ simulators, the dielectric substrate is extended to infinity in space [1]. Any abrupt transition from substrate to air in the real antenna will be ignored. Therefore, any surface wave bounced back from the edges of the board will not be considered, thus introducing errors into the calculation of the resonant frequency $[7,5]$. Infinite substrate simplifies the calculation and allows the reuse of the substrate data, resulting in much shorter simulation time. A limitation of the infinite substrate is the incorrect modeling of the far field along the dielectric plane as shown in Figure 2. The 3D
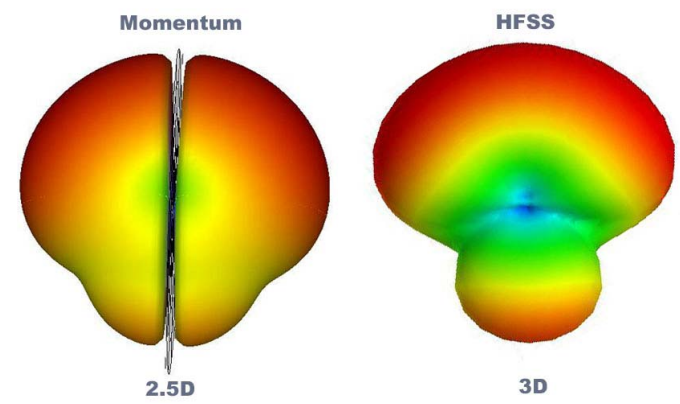

Figure 2: 2.5D (left) vs. 3D (right) far-field modeling

simulator considers the effects of the surface wave bounced back from the edges considered during the simulation. That contributes to the simulation matching more precisely the measurements. Field strength measured along the dielectric plane on the fabricated antenna was eventually found to match closely the results of the 3D HFSS simulations.

\section{PRINTED $\lambda / 2$ DIPOLE DESIGN}

The printed half-wave dipole design is derived from $[4,3$, 9 ], where the major modification is in the replacement of the 
rectangular shaped dipole arms by the metal plates on the opposite face of the PCB to form the "end-loaded" dipole. The goal is to reduce the size to less than a half-wavelength, and save the PCB area. This design concept is borrowed from the capacitor-plate antenna. Since a dipole becomes inductive when it is shorter than half a wavelength [11], the capacitive plates are used to cancel this inductance. Since the capacitance is dependent on the dimensions of the plates, by changing the plates' dimensions, the resonant frequency can be tuned. In our case, the area of $1.8 \mathrm{~cm} \mathrm{X} 3.9 \mathrm{~cm}$ is $60 \%$ smaller than the original design [3]. In addition, the gain and the radiation pattern remains unchanged. Figure 3 shows the predicted $S_{11}$ response of this antenna. The sec-

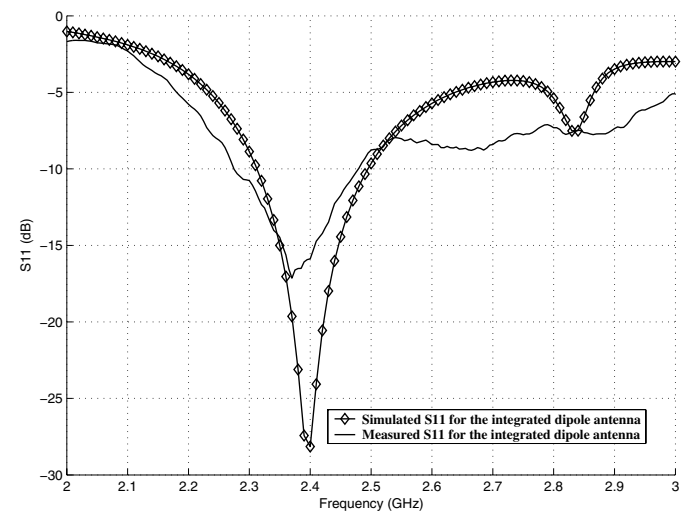

Figure 3: HFSS-simulated and measured $S_{11}$ response of the half-wave dipole antenna

ond major characteristic of this design is the simple via-hole balun that avoids differential inputs and allows the use of a microstrip feed line. The usage of the $50 \Omega$ feed line eliminates the complex differential measurements that usually require costly equipment or calibrated balun, reducing the testing time. Notice that an alternative is to feed a balanced dipole directly from the differential RF-port of the transceiver [2]. This will save the cost of the balun circuit composed of lumped components. However, this will require differential test setups. Thus, the compromise must be made between the cost of manufacturing and that of design and test.

\subsection{System-Level Effects - Feed Network}

Although the antenna can work alone perfectly, the analysis is not complete without the presence of the transceiver and other circuit elements of the wireless node. Therefore, the effect of the circuit components in the near field of the antenna [11, p.30] must be analyzed.

To fulfill the routing requirements and maximize cost savings, the Chipcon CC2420 transceiver is built on a two-layer PCB. The top layer is used for signal and power routing. The bottom layer is used as the ground plane and must be kept free of large holes when traces must be routed on it. Like most multi-layered boards, these two layers can act as a microstrip patch antenna and can generate undesired radiation $[6,12,13]$. To avoid this problem, the filled areas of the top layer are stitched with the bottom ground plane using vias. Those will suppress the parallel mode that may exist between the two layers. Without them, part of the power fed to the antenna is launched between the top and bottom planes. This will result in an extra resonance that distort the passband of the antenna [10]. The radiation pattern will also be affected. The effectiveness of the vias depends on their placements on the PCB. They must be placed near the edges of the two conducting layers to suppress parallel-plate modes and fringing fields of the unwanted patch antenna [6]. The vias should be placed apart less than half wavelength to avoid resonance [12]. The resulting PCB model is shown in figure 4 . Note that this PCB model was simplified to accelerate HFSS simulations by removing digital traces as seen in comparison with the photograph. The complete PCB database is maintained in the Mentor Expedition PCB tool set and is exported to HFSS for simulations as part of the design iterations.
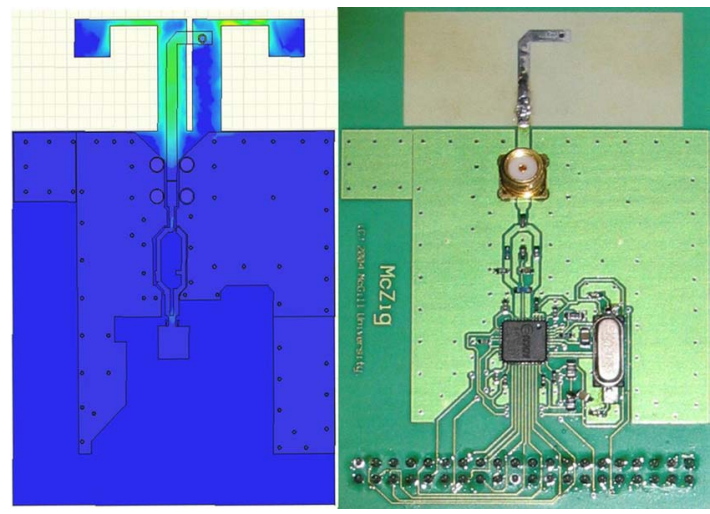

Figure 4: Prototype simulation model showing peak currents (left) and photograph (right)

From the simulation results, the major effect of the feed network is the alteration of the radiation pattern. From image theory, the opposite side of the ground plane will have stronger field due to reflections [11]. This is clearly shown in the simulated radiation pattern. The pattern still looks like the traditional "doughnut" shape of the dipole pattern but has an asymmetry of $8 \mathrm{~dB}$ as shown in Figure 5 for angles of $30^{\circ}$ and $150^{\circ}$.

The antenna analysis and measurements are summarized in Table 2 .

Table 2: Prototype Antenna Properties

\begin{tabular}{|c|c|c|}
\hline Property & Value & Method \\
\hline Peak Gain & 2.64 & Simulation \\
\hline Efficiency & $99.1 \%$ & Simulation \\
\hline Center Frequency & $2.400 \mathrm{GHz}$ & Simulation \\
\hline Center Frequency & $2.368 \mathrm{GHz}$ & Lab Measure \\
\hline Bandwidth (2:1 VSWR) & $200 \mathrm{MHz}$ & Simulation \\
\hline Bandwidth (2:1 VSWR) & $290 \mathrm{MHz}$ & Lab Measure \\
\hline
\end{tabular}

\section{MEASUREMENT RESULTS}

Using the outlined design methodology, a IEEE 802.15.4 WLAN node was designed and manufactured to validate its operation in a working system. The board was fabricated on RO4350B (see Figure 4) and its performance was measured in the lab. Antenna admittance $S_{11}$ was measured by 


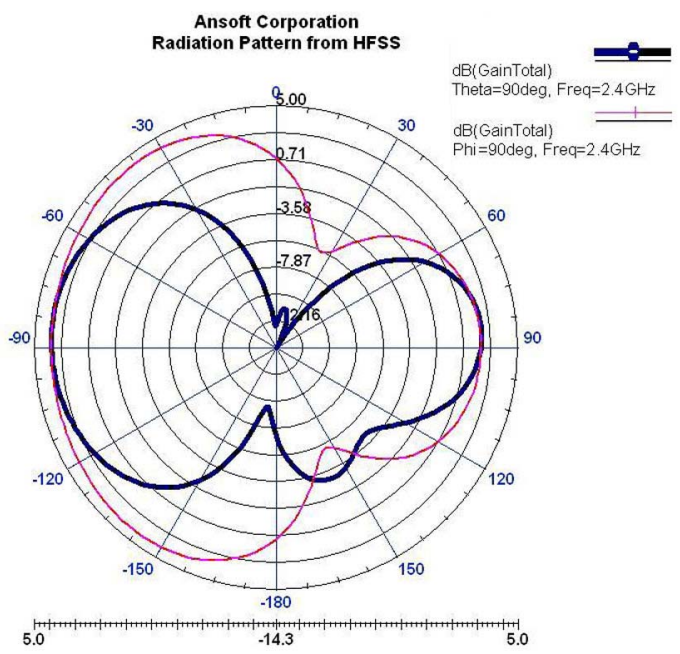

Figure 5: Simulated E-Field strength on the PCB plane (bold) and perpendicular (thin)

connecting the feed line of the antenna to a HP 8593E Vector Network Analyzer using the SMA connector on the prototype. The center frequency simulation matches to within $1.8 \%$ the HFSS model in Figure 3. The discrepancy between them originate from lack of accuracy in low-admittance measurement without an RF anechoic chamber and distortion from the test connector.

Note that a solder mask opening was created around the antenna feed point in order to avoid a second dielectric layer that could affect the test results. Figure 4 shows both the HFSS model (the left half) and the final PCB. Unlike the model, tapering out of the ground plane surrounding the microstrip line is not present on the PCB. This tapering avoids a discontinuity in the trace impedance at it exits the ground plane and slightly improves the matching of the antenna.

The transmitter board was also tested in a complete system deployed in a convention center environment. During prolonged use, it was found that the board allows reliable transmission $(<1 \%$ Packet Error Rate) at ranges exceeding 60 meters, and through 4 concrete walls. In this prototype the balun circuit was borrowed from the CC2420 reference design[2]. Only the antenna and feed network were analyzed using the electromagnetic simulation tools. The range can be further extended by integrating the balun circuit in the RF model, allowing for its optimization.

The methodology presented in this paper was also successfully applied to quarter-wave monopoles, folded monopoles and inverted-F classes of antennas. S-Parameter measurements agreed with simulation models to the same extent as the sample design presented in this paper.

\section{CONCLUSIONS}

This paper presented the methodology needed to realize printed antennas on a PCB substrate predictably and without extensive lab testing over numerous PCB iterations. The interference with the board electronics was modeled, and effectively avoided through a combination of design techniques and modeling interchange with the basic PCB design flow.
While specialized RF dielectric materials such as RO4350B ensure predictability, the same design methodology can be used with the most elementary PCB materials in large quantities if the slightly lower efficiency is not a concern or if the PCB material properties can be kept uniform across manufacturing runs. Finally, to get optimal performance, every element carrying RF signals should undergo full-wave EM modeling.

In the authors opinion, the methodology can be applied to the design of any resonnant antenna operating in the 1 to $6 \mathrm{GHz}$ range. Beyond $6 \mathrm{GHz}$, the thin antenna elements will lead to larger losses in the dielectric and feed lines.

\section{ACKNOWLEDGMENTS}

The authors would like to thank Dr. Zoya Popović from University of Colorado at Boulder for providing insights during the initial phases of this project. We would also like to acknowledge the contribution of Rogers Corporation in this project in providing free samples of RF PCB laminates, the Canadian Microelectronic Corporation for providing test equipment, Agilent Technologies and Ansoft Corporation for their contribution through academic licensing of their EDA tools. Part of this work was funded through the "Fonds Québécois de la recherche sur la nature et les technologies".

\section{REFERENCES}

[1] Agilent. Advanced design system 2003a documentation. Product documentation, Agilent Technologies, 2003.

[2] Chipcon. SmartRF CC2420 preliminary datasheet (rev 1.2). Datasheet, Chipcon AS, Gaustadalléen 21 NO-0349, Oslo, NORWAY, 2004.

[3] H.-R. Chuang and L.-C. Kuo. 3-D FDTD design analysis of a 2.4-ghz polarization-diversity printed dipole antenna with integrated balun and polarization-switching circuit for WLAN and wireless communication applications. IEEE Trans. Microwave Theory Tech., 51(2), February 2003.

[4] D. Edward and D. Rees. A broadband printed dipole with integrated balun. Microwave J., pages 339-344, May 1987.

[5] M. Gimersky and J. Bornemann. A modified method-of-moments technique for the full-wave analysis of imperfect conductors on lossy and finite-extent substrates. IEEE MTT-S Int. Microwave Symp. Digest, 2:715-718, June 1996.

[6] W. H. Haydl. On the use of vias in conductor-backed coplanar circuits. IEEE Trans. Microwave Theory Tech., 50:1571-1577, June 2002

[7] Y.-H. Pang and R.-B. Wu. Analysis of microstrip antennas with finite-sized substrate. IEEE AP-S Int. Symp., 2:814-817, July 2001.

[8] T. S. Rappaport. Wireless Communications: Principles and Practice. Prentice Hall, Upper Saddle River, New Jersey, 2nd edition, 2002

[9] M. Scott. A printed dipole for wide-scanning array application. $11^{\text {th }}$ Int. Conf. Antennas and Propagation, 1:37-40, 17-20 April 2001.

[10] C. Soras, M. Karaboikis, G. Tsachtsiris, and V. Mikios. Analysis and design of an inverted-F antenna printed on a PCMCIA card for the $2.4 \mathrm{GHz}$ ISM card. IEEE Antenna's and Propagation Magazine, 44:37-44, February 2002.

[11] W. L. Stutzman and G. A. Thiele. Antenna Theory and Design. Wiley, second edition, 1997.

[12] F. Xiao, K. Murano, and Y. Kami. The use of via holes for controlling the crosstalk of non-parallel microstrip lines on PCBs. 2002 IEEE Int. Symp. On Electromagnetic Compatibility, 2:633-638, August 2002.

[13] X. Ye, D. M. Hockanson, M. Li, Y. Ren, W. Cui, J. L. Drewniak, and R. E. DuBroff. EMI Mitigation with multilayer power-bus stacks and via stitching of reference planes. IEEE Trans. Electromagnetic Compatibility, 43:538-548, November 2001. 